\title{
Mechanism Confirmation of Organofunctional Silanes Modified Water Glass/Polyurethane-Urea Composites for Remarkably Enhanced Mechanical Properties
}

\section{Yuntao Liang}

State Key Laboratory of Coal Mine Safety Technology

\section{Ao Gao}

Shandong University of Science and Technology

\section{Yong Sun}

State Key Laboratory of Coal Mine Safety Technology

\section{Fuchao Tian}

State Key Laboratory of Coal Mine Safety Technology

\section{Weili Sun}

State Key Laboratory of Coal Mine Safety Technology

Wei Lu

Shandong University of Science and Technology

Zhenglong He ( $\nabla$ hzl_safety@sdust.edu.cn )

Shandong University of Science and Technology

\section{Research Article}

Keywords: Polyurethane-urea, Water glass, Mechanism analysis, Mechanical properties, Organofunctional silanes

Posted Date: March 8th, 2021

DOI: https://doi.org/10.21203/rs.3.rs-274505/v1

License: (c) (1) This work is licensed under a Creative Commons Attribution 4.0 International License. Read Full License 


\section{Abstract}

Hybrid reinforced water glass/polyurethane-urea (WG/PU) composites mainly derived from low-cost WG and polyisocyanate are produced in the presence of 3-chloropropyltrimethoxysilane (CTS). The wettability of WG on PU substrate surface is significantly improved as CTS content increases from $0.0 \mathrm{wt} \%$ to 3.5 $w t \%$. Furthermore, with $2.5 \mathrm{wt} \%$ of CTS optimal addition, the fracture surface morphology and elemental composition of the resulting WG/PU composites are characterized, as well as mechanical properties, chemical structure and thermal properties. The results indicate that the CTS forms multiple physical and chemical interactions with the WG/PU composites to induce an optimized organic-inorganic hybrid network structure thus achieving simultaneous improvement of compressive strength, flexural strength, flexural modulus and fracture toughness of the WG/PU composites, with the improvement of $12.9 \%$, $6.6 \%, 17.5 \%$ and $9.7 \%$, respectively. Moreover, the CTS in the WG/PU composites has a significant influence on the curing process of the WG/PU composites by accelerating the rate of mass and heat transfer. Additionally, a reasonable mechanism explanation for CTS modified WG/PU composites is confirmed.

\section{Introduction}

Polyurethane-urea is widely used as grouting materials in the mining industry ${ }^{1-4}$, building foundation reinforcement ${ }^{5-7}$ and road maintenance ${ }^{8-12}$, because of its excellent characteristics in terms of efficient performance and easy operation. However, it is found that the high cost, high heat release and flammability of polyurethane-urea production limit its application. Especially, these disadvantages are fatal and prohibit in the field of coal mining. To overcome the disadvantages, inorganic modification is a promising strategy in improving the performance of the composites ${ }^{13,14}$. Hybrid organic/inorganic composites made from polyisocyanate and water glass possess low cost, good permeability, low heat release and fire resistance, but have poor mechanical properties due to the interface incompatibility between organic and inorganic phase ${ }^{15}$. Various new interface modification additives have been used as an emulsifier for the improvement of the WG/PU composites performance, such as phosphate ${ }^{16}$, vinyl ester ${ }^{17}$, melamineformaldehyde ${ }^{18}$, epoxy ${ }^{19}$. Although several additives have shown effective mechanical reinforcements in WG/PU composites, it is difficult to simultaneously improve the compressive strength, flexural strength, flexural modulus and the fracture toughness of the WG/PU composites. Later, $\mathrm{He}^{2}$ and Kopietz ${ }^{1}$ proposed that the mechanical properties (e.g. compressive, fracture, flexural and dynamic mechanical properties) of WG/PU composites could be dramatically improved using the organofunctional silanes. Although it is confirmed that the particle dispersion and cavitation can promote the strength and toughness of the WG/PU composites, the mechanism of the organofunctional silanes act on the WG/PU composites remains unclear. Moreover, previous studies have shown a debonding cavitation between organic phase and inorganic phase due to the volume shrinkage of the inorganic phase during the hydrogel-xerogel transition. The introduction of the organofunctional silanes in the WG/PU composites can not promote the interfacial bonding between the inorganic phase and the organic 
phase. Therefore, understanding the behavior mechanism of the organofunctional silanes in the WG/PU composites to achieve WG/PU composites with good reinforcement performances is highly desirable.

Herein, we report the successful fabrication of organic-inorganic efficient performance and easy operation WG/PU composites by the organofunctional silanes 3-chloropropyltrimethoxysilane (CTS). The effect of the CTS on the wettability of the WG and the compressive strength of the WG/PU composites are investigated. Meantime, it also evaluates the flexural and fracture properties of the WG/PU composites with the optimum dosage of CTS. Furthermore, the curing mechanism of the WG/PU composites by introducing CTS is determined by using Scanning electron microscopy (SEM), Energy dispersive spectrometry (EDS), Fourier transform infrared (FTIR), Diffuse reflectance infrared Fourier transform spectroscopy (DRIFTS), Thermogravimetric analysis (TGA) and Differential scanning calorimetry (DSC) measurements.

\section{Experiments \\ 2.1 Materials}

The polyisocyanate (PM-200, isocyanate content: 30.5 $32 \mathrm{wt} \%$ ) is kindly supplied by Yantai Wanhua Polyurethanes Co., Ltd. The polyether polyol with molecular weights of 2000 (GE-220) and chlorinated paraffin-52 are purchased from Shanghai Gaoqiao Petrochemical Co., Ltd. Water glass (WG) is purchased from Shandong Hongquan Chemical try Co., Ltd. 3-chloropropyltrimethoxysilane (CTS, 98\%) and cyclohexylamine $(98 \%)$ are obtained from Aladdin.

\subsection{Sample Preparation}

WG/PU composites are prepared via a conventional mixing and curing procedures of two components. Briefly, 30 parts $(\mathrm{w} / \mathrm{w})$ of $W G$ and a certain amount of CTS are placed in a $500 \mathrm{~mL}$ plastic cup and homogenized at $300 \mathrm{rpm}$ for 30 min using a mechanical stirrer. 1 part $(\mathrm{w} / \mathrm{w})$ of cyclohexylamine is subsequently added to the WG systems and are mixed further for $4 \mathrm{~min}$ at $500 \mathrm{rpm}$, and then the appropriate amount of the organic component such as PM-200, chlorinated paraffin-52 and GE-220 are added and homogenized again for $1 \mathrm{~min}$ at $300 \mathrm{rpm}$. After mixing process, the WG/PU composites are synthesized and cured in different steel molds at ambient condition.

\subsection{Sample characterization}

The wettability alteration behavior for the WG/CTS system is characterized by the contact angle between the modified WG and the organic component. A droplet of WG with different CTS contents is placed on the cured organic component surface. Images of the equilibrium contact angle of the mixed WG/CTS droplet are recorded by the SL200B machine (KINO Industry Co., Ltd., USA). The mechanical tests of the compressive, flexural and fracture properties of the WG/PU composites are performed on an electron omnipotence experiment machine (SANS-CMT6503, Shenzhen Sans Testing Machine Co., China) at ambient conditions according to GB/T 1041-1992, EN 63 and ISO-13586-1, respectively. The testing results are reported as the average of at least three measurements. The fracture surface morphology and 
the elemental composition of the WG/PU composites are analyzed by a scanning electron microscope (SEM, 2800B, KYKY, China) with an energy dispersive spectrometer (EDS, QX200, Bruker, Germany).

Bruker Tensor 27 FTIR Spectrometer (Bruker, Germany) is used for the quantitative analysis ${ }^{20}$ of isocyanate (NCO) peak intensity in the WG/PU composites under different curing time. Diffuse reflectance infrared Fourier transform spectroscopy (DRIFTS) is recorded for the fully cured modified and non-modified WG/PU composites using a Bruker Tensor 27 instrument equipped with a MCT detector. Thermogravimetric analysis (TGA) is performed using a PerkinElmer STA6000 thermal analyzer at $10^{\circ} \mathrm{C} \cdot \mathrm{min}^{-1}$ heating rate from $30^{\circ} \mathrm{C}$ to $600^{\circ} \mathrm{C}$ under a nitrogen atmosphere with flow rate of $30 \mathrm{~mL} \cdot \mathrm{min}^{-1}$. Differential scanning calorimetry (DSC) analysis is conducted on a Mettler-Toledo DSC823e system to study the thermal properties of the modified and non-modified WG/PU composites under a nitrogen atmosphere at a heating rate of $10^{\circ} \mathrm{C} \cdot \mathrm{min}^{-1}$ within the temperature's range from $30^{\circ} \mathrm{C}$ to $250^{\circ} \mathrm{C}$.

\section{Results And Discussion}

\subsection{Synthesis mechanism of CTS modified WG/PU composites}

Scheme 1 schematically shows the primary chemical reactions route that occurs during the WG/PU composites preparation and the CTS effect. The organic component and the modified WG are mixed using a two-blade paddle mixer. The achieved homogeneous mixed slurry is poured into the mold and curied until theexpected WG/PU composites is achieved (see Scheme 1a). During the process of mixing, the hydrolyzed CTS is oriented at the interface between the organic phase and the inorganic phase, thus forming a smaller and more uniformed inorganic dispersion system by the reduction of the organicinorganic interfacial tension (see Scheme $1 b$ and $d$ ). This benefits the acceleration of the curing process and the better dispersion of the stress. Thus, the mechanical properties of the composites are further improved. During the following curing of WG/PU composites, multifunctional polyisocyanate is reacted with the water from the WG, leading to a highly cross-linking network of formed polyurea by urea units in the organic phase (see Scheme 1c). More water consumption produces more carbon dioxide into WG, which in turn produces more sodium carbonate, leading to gelation of the WG in the inorganic phase. Water continuously enters the organic phase from the inorganic phase, while the diffusion path of the produced carbon dioxide is opposite. The directional mass transfer of water and carbon dioxide together realizes the hardening of the WG to the polysilicate and the cross-linking polymerization of the organic phase, and induces the solidification of of the organic phase and the inorganic phase eventually. Moreover, a large amount of reaction heat from the amine-isocyanate chemistry will also accelerate this curing process. In addition, the lipophilic group can make part of CTS get into the organic phase welldistributed. Subsequently, the reactive lipophilic groups of the CTS as hard segment will bind with the crosslinking point (e.g. the NCO and urea groups) of the organic phase, forming a chemical bond. Therefore, it is precisely because of multiple physical and chemical interactions between the CTS and the WG/PU composites that the WG/PU composites possess the outstanding mechanical properties. 
To achieve high mechanical performance of the composite, interface properties between the organic and the inorganic phases need to be improved. Figure 1 shows the static contact angle of the modified WG on the cured organic component surface. Pure WG presents a contact angle of $117.49^{\circ}$, whereas by modifying it is gradually reduced from $117.49^{\circ}$ to $77.73^{\circ}$. An improvement of more than $33 \%$ is observed in wettability of WG on the cured organic component surface. It is probably ascribed to the presence of lipophilic CTS on the WG surface. This result is confirmed by modification, which makes WG more lipophilic. This improved wetting property is beneficial to the refinement and uniform distribution of the WG in PU matrix and improves the strength of the WG/PU composites.

\subsection{Mechanical properties of WG/PU composites}

Figure 2 shows that the compressive strength obtained for the WG/PU composites with the CTS content of $0.0 \mathrm{wt} \%, 0.5 \mathrm{wt} \%, 1.5 \mathrm{wt} \%, 2.5 \mathrm{wt} \%$ and $3.5 \mathrm{wt} \%$. The CTS is observed to enhance the compressive strength of the WG/PU composites significantly. Excellent wettability allows maximum inorganic phase refinement. Accordingly, the curing process of the WG/PU composites becomes faster and more complete. Therefore, the compressive strength of the WG/PU composites varies from 55.07 MPa to 62.19 $\mathrm{MPa}$ with the increase of the CTS content from $0.0 \mathrm{wt} \%$ to $2.5 \mathrm{wt} \%$. As CTS content is greater than 2.5 $\mathrm{wt} \%$, a peak is reached for the WG/PU composites, showing a slight decrease in compressive strength thereafter. This is probably due to the arising interface saturation with the CTS in the WG/PU composites. The outcome therefore indicates that the interface saturation between the inorganic phase and organic phase may start as early as the $2.5 \mathrm{wt} \%$ CTS content range, which is adequate to reach high compressive performance.

To further understand the mechanical properties of the WG/PU composites, the flexural strength, flexural modulus and fracture toughness of the WG/PU composites with $2.5 \%$ of CTS optimal addition and without CTS are examined respectively, as shown in Figure 3. From Figure 3, it is observed that $2.5 \mathrm{wt} \%$ of the CTS appropriately improves the average flexural strength of the WG/PU composites from $39.3 \mathrm{MPa}$ to 41.9 MPa. However, the $17.5 \%$ and $9.7 \%$ enhancement in flexural modulus and fracture toughness are achieved from $2.5 \mathrm{wt} \%$ CTS modified the WG/PU composites, respectively. This may be attributed to the active crosslinking effect of the CTS. The CTS can enter and form strong chemical interactions with the organic phase of the WG/PU composites and further strengthen the interior crosslinking structure of the organic phase, thus facilitating the improvement of strength and toughness for the WG/PU composites. Therefore, $2.5 \mathrm{wt} \%$ of CTS will be used as the designated amount of modified WG/PU composites. The obtained WG/PU composites will be used for the fracture surface morphology, elemental composition, chemical structure and thermal properties analysis.

\subsection{SEM-EDS analysis of WG/PU composites}

In order to understand constitutes of the WG/PU composites and further explore how the CTS induces physical and chemical interactions to enhance the mechanical performance of the WG/PU composites, SEM and EDS are used together to observe the fracture surface morphology and analyze the elemental composition and content in the WG/PU composites. The SEM images show the spherical dispersed 
phases embedded in the the continuous phase. Moreover, the continuous phases exhibit many domeshaped cavities throughout the fractured surface of the compsites. It is obvious that there is insufficient interfacial bond strength between the continuous phase and the dispersed phase. The EDS analyses show that the continuous phases consist of C (65.54 wt\%), O (11.38wt\%), Cl (13.17 wt\%) and C (70.11 wt\%), $\mathrm{O}$ (13.23 wt\%), $\mathrm{Cl}$ (12.98 wt\%) in the non-modified and modified WG/PU composites, respectively. It reveals the continuous phase originated from the organic component. On the other hand, the main elements of the dispersed phase are Si (32.15 wt\%), O (44.96 wt\%), Na (12.57 wt\%), C (10.33 wt\%) and Si (30.10 wt\%), O (46.65 wt\%), Na (10.04 wt\%), C (13.21 wt\%) in the non-modified and modified WG/PU composites, respectively. The results therefore prove that the dispersed phase originates from the WG.

As shown in Figure $4 \mathrm{~A}(\mathrm{a})$ and $\mathrm{B}(\mathrm{a})$, compared with the non-modified samples, $2.17 \mathrm{wt} \%$ of the Si element appears in the continuous phase of the modified sample. This phenomenon illustrates that the CTS will migrate into the organic phase in the modified WG/PU composites. Figure $4 A(b)$ and $B(b)$ show that the element content of C (42.99 wt\%), Cl (24.90 wt\%) and Si (2.78 wt\%) on the cavity surface of the modified composites are much higher than that (C (21.09 wt\%), $\mathrm{Cl}(10.75 \mathrm{wt} \%)$ and Si (trace)) of the non-modified composites. This confirms that the CTS is widely distributed at the interface between the organic phase and the inorganic phase. Furthermore, it is found that $\mathrm{C}$ and $\mathrm{Cl}$ elements are greatly increased on the cavity inner surface of the modified WG/PU composite. This demonstrates that the introduction of CTS improves the interface compatibility and affinity between organic and inorganic phases. In addition, it can be clearly seen that the $\mathrm{O}(46.65 \mathrm{wt} \%)$ and C (13.21 wt\%) content on the dispersed phase of the modified sample are higher than that $(\mathrm{O}(44.96 \mathrm{wt} \%)$ and $\mathrm{C}(10.33 \mathrm{wt} \%))$ of the non-modified sample (see Figure $5 \mathrm{~A}(\mathrm{c})$ and $\mathrm{B}(\mathrm{c})$ ). This increment is primarily attributable to higher $\mathrm{CO}_{2}$ accumulation rates at the inorganic-organic phase interface caused by the rapid cross-linking polymerization of the organic phase in the modified WG/PU composites.

In order to confirm the influence of CTS on the distribution and morphology of WG in the PU matrix. The cross-sectional SEM images of the non-modified and modified WG/PU composites are shown in Figure $5 a$ and $b$. The SEM images confirm that the polysilicate particles are individually dispersed in the PU matrix. Moreover, the SEM images show that the polysilicate particle size and dome-shaped cavities have become smaller and more uniform in the modified WG/PU composites due to the addition of CTS, which is responsible for the relatively high mechanical strength and toughtness of WG/PU composites. In addition, Figure $5 \mathrm{c}$ and d directly show the size distribution histograms of the polysilicate particles of the non-modified and modified samples. The size of polysilicate particle varies from $0 \mu \mathrm{m}$ to $60 \mu \mathrm{m}$, and 0 $\mu \mathrm{m}$ to $24 \mu \mathrm{m}$ for the non-modified and modified samples, respectively. Moreover, the mean size of polysilicate particle for the non-modified and modified samples are also reduced from $11.3 \mu \mathrm{m}$ to $5.7 \mu \mathrm{m}$. The results demonstrate that the CTS has excellent emulsification ability. Additionally, $4.4 \%$ of the polysilicate particles with a scale between $24 \mu \mathrm{m}$ to $60 \mu \mathrm{m}$ are present in non-modified samples. Increasing the size of polysilicate particles leads to larger cavities formation. These particles and cavities as defects can easily cause stress concentration, which deteriorates the mechanical properties of the 
WG/PU composites. The micro-structural analysis of WG/PU composites ensures the excellent emulsifying ability of the CTS, which is consistent with the results of mechanical performance testing.

\subsection{IR spectra of WG/PU composites}

The intensity of the NCO peak is a very important parameter, expressing the degree of curing of WG/PU composites. The strong absorption peak at $2270 \mathrm{~cm}^{-1}$ corresponds to the NCO group stretching in the WG/PU composites. As shown in Figure 6, whether modified or not, the NCO peak intensity of the WG/PU composites gradually decreases as the the curing time increases from $0.5 \mathrm{~d}$ to $28 \mathrm{~d}$. This implies that NCO groups in the organic phase are gradually consumed by the diffused water from the inorganic phase during the curing process of the WG/PU composites. Moreover, by comparison, the NCO peak intensity of the modified WG/PU composites is weaker than that of the non-modified WG/PU composites under the same curing time. This difference indicates that the curing process of the WG/PU composites can be accelerated by the addition of CTS due to the intensity of the peak decreases progressively as the curing rate increases. In addition, in comparison with the non-modified WG/PU composites, the absorption peak of NCO groups exhibited by the modified WG/PU composites decreases after fully curing. This is due to the fact that the CTS enters the organic phase as the additional crosslinking points form chemical interactions with NCO groups in the organic phase.

The DRIFTS spectrum obtained for the modified WG/PU composites is compared with those for the nonmodified WG/PU composites in Figure 7, which shows that the nearly identical spectrum curves of modified and non-modified WG/PU composites. This indicates that the addition of CTS has no obvious effect on the change of the chemical structure of the WG/PU composites. The absorption peaks at 3280$3360 \mathrm{~cm}^{-1}$ and $1680 \mathrm{~cm}^{-1}$ might contribute to amide $\mathrm{N}-\mathrm{H}$ stretching peak and the urea carbonyl groups $(\mathrm{C}=0)$, which is the essential structures for polyurea from the reaction between NCO groups and water molecules. However, the characteristic peak of $\mathrm{NCO}$ at $2267 \mathrm{~cm}^{-1}$ is still observed, indicating that there might be a small amount of residual NCO in the WG/PU composites after fully curing.

\subsection{Thermo gravimetric analysis of WG/PU composites}

In order to fully evaluate the effect of CTS on thermal properties of WG/PU composites, we investigate the course of thermal decomposition of WG and WG/PU composites before and after modification (see Figure 8a). In the temperature range from $30^{\circ} \mathrm{C}$ to $250^{\circ} \mathrm{C}$ the free water and the crystallization water are lost from the WG, with maximu rate at $80^{\circ} \mathrm{C}$ and $132^{\circ} \mathrm{C}$, resepcetively. Similarly, the DTG peaks of WG/PU composites within $50^{\circ} \mathrm{C}-250^{\circ} \mathrm{C}$ are due to elimination of water and a small amount of low molecular weight organics release. Between $50^{\circ} \mathrm{C}$ and $178{ }^{\circ} \mathrm{C}$, an obvious reduction in mass loss rate for modified WG/PU composites compares to non-modified WG/PU composites. It results from the fact the dispersion of WG particles in the PU matrix is higher and finer than in case of non-modified WG/PU compsites, therefore, the water from the WG can be more easily consumed. Moreover, a strong degradation peak is detected between $178^{\circ} \mathrm{C}$ and $220^{\circ} \mathrm{C}$, which should be linked with the evaporation of the CTS present at the inorganic-organic phase interface of the modified WG/PU compsites. Besides, 
three other typical weight loss steps are observed: the first and second mass loss occurring at $330^{\circ} \mathrm{C}$ and $412^{\circ} \mathrm{C}$ can be attributed to the degradation of hard and soft segment, respectively ${ }^{21}$. The third weight loss in the range of $480^{\circ} \mathrm{C}-550^{\circ} \mathrm{C}$ might be due to the degradation of the rest of the organic phase $\mathrm{e}^{22}$. In this step, the weight loss in the temperature range of $490^{\circ} \mathrm{C}-506^{\circ} \mathrm{C}$ seems to be ascribed to the decomposition of the $\mathrm{CTS}^{23}$. Moreover, the decomposition temperature is notably higher than the boiling point $\left(195^{\circ} \mathrm{C}\right)$ of the CTS becasue the CTS forms strong chemical bonds with the organic phase.

Figure $8 \mathrm{~b}$ displays the DSC thermograms of the non-modified and modified WG/PU composites. The DSC thermograms shows that there is a complex structure in the WG/PU composites. It will not clearly discern the glass transition temperature $\left(T_{g}\right)$ of the WG/PU composites. This phenomenon is attributed to that $\mathrm{Tg}$ is superimposed to thermal evaporation and degradation ${ }^{24}$. In the non-modified and modified WG/PU composites, the broad endothermic peak at $120^{\circ} \mathrm{C}-210{ }^{\circ} \mathrm{C}$ is ascribed to the endothermic melting peak of hard segment $\left(T_{m, h s}\right)$. The addition of CTS has a notable effect on the $T_{m}$, hs value of the WG/PU composites, which can be observed from the incremental change in $T_{m}$, hs values. It mainly attributed to the increasing of hard segments contents and crosslinking density by the CTS as the active hard segment corsslinker chemical links with the organic phase. A strong endothermic peak at $190{ }^{\circ} \mathrm{C}$ superimposes to the $T_{m, h s}$ and the result confirms that the evaporation of the CTS because this temperature is close to the boiling point of free CTS. It also indicates that partially CTS is not bonded to the dispersed or continuous phases in the WG/PU composite, which is in good agreement with the TGA results.

\section{Conclusions}

Highly mechanical properties of WG/PU composites made from multifunctional polyisocyanate and lowcost WG are successfully realized by introducing the organofunctional silanes CTS via the simple mixing. The addition of the CTS realizes the refinement and uniform distribution of the inorganic phase, and the acceleration of the curing process in the WG/PU composites. Meanwhile, the CTS as an active hard segment crosslinker forms chemical interactions with iscyanate groups in the organic phase. These effects will be beneficial to induce an optimized organic-inorganic hybrid network structure, thereby achieving simultaneous improvement of compressive strength, flexural strength, flexural modulus and fracture toughness of the WG/PU composites. Moreover, the rapid curing of the WG/PU composites composed of the hardening of the WG and the intermolecular crosslinks of the PU monomers is achieved by mass and heat transfer between the inorganic phase and the organic phase. The resultant work will provide the possibility to prepare high-performance WG/PU composites for more potential engineering application fields.

\section{Declarations}

Acknowledgments 
The authors gratefully acknowledge financial support from the National Key R \& D Program of China (No. 2018YFC0807900, 2018YFC0807906), the National Natural Science Foundation of China (No. 51574279, 52004148), Taishan Scholar Talent Team Support Plan for Advantaged \& Unique Discipline Areas, the Natural Science Foundation of Shandong Province (ZR2020QE127) and the Open Project Funding (2020KF-23-05) of the State Key Laboratory of Coal Mine Safety Technology (CCTEG Shengyang Research Institute).

\section{References}

1. Kopietz, M., Wetzel, B. \& Friedrich, K. Flexural and fracture mechanical properties of in situ particulate reinforced organomineral hybrid resins modified by organofunctional silanes. Composites Science and Technology. 174, 169-175 https://doi.org/10.1016/j.compscitech.2019.02.013 (2019).

2. He, Z. et al. Effect of silane treatment on the mechanical properties of polyurethane/water glass grouting materials. Construction and Building Materials. 116, 110-120 https://doi.org/10.1016/j.conbuildmat.2016.04.112 (2016).

3. Yang, Z. et al. Polyglycerol-based organic-inorganic hybrid adhesive with high early strength. Mater. Design. 117, 1-6 https://doi.org/10.1016/j.matdes.2016.12.069 (2017).

4. Liu, Z., Wang, W., Cheng, W., Yang, H. \& Zhao, D. Study on the seepage characteristics of coal based on the Kozeny-Carman equation and nuclear magnetic resonance experiment. Fuel. 266, 117088 https://doi.org/10.1016/j.fuel.2020.117088 (2020).

5. Buzzi, O., Fityus, S., Sasaki, Y. \& Sloan, S. Structure and properties of expanding polyurethane foam in the context of foundation remediation in expansive soil. Mechanics of Materials. 40, 1012-1021 https://doi.org/10.1016/j.mechmat.2008.07.002 (2008).

6. Liu, K. et al. The study on compressive mechanical properties of rigid polyurethane grout materials with different densities. Construction and Building Materials. 206, 270-278 https://doi.org/10.1016/j.conbuildmat.2019.02.012 (2019).

7. Saleh, S., Yunus, N. Z. M., Ahmad, K. \& Ali, N. Improving the strength of weak soil using polyurethane grouts: A review. Construction and Building Materials. 202, 738-752 https://doi.org/10.1016/j.conbuildmat.2019.01.048 (2019).

8. Huang, H., Pang, H., Huang, J., Zhao, H. \& Liao, B. Synthesis and characterization of ground glass fiber reinforced polyurethane-based polymer concrete as a cementitious runway repair material. Construction and Building Materials. 242, https://doi.org/10.1016/j.conbuildmat.2019.117221 (2020).

9. Saleh, S., Ahmad, K., Mohd Yunus, N. Z. \& Hezmi, M. A. Evaluating the toxicity of polyurethane during marine clay stabilisation. Environ Sci Pollut Res Int. 27, 21252-21259 https://doi.org/10.1007/s11356-020-08549-y (2020).

10. Wan, Z., Bian, X., Li, S., Chen, Y. \& Cui, Y. Remediation of mud pumping in ballastless high-speed railway using polyurethane chemical injection. Construction and Building Materials. 259, https://doi.org/10.1016/j.conbuildmat.2020.120401 (2020). 
11. Yang, Z. et al. Fabrication and performance of a polyurethane hybrid composite with waste red mud. Polym. Compos. 40, 2424-2431 https://doi.org/10.1002/pc.25107 (2018).

12. Yang, Z. et al. Flexible and stretchable polyurethane/waterglass grouting material. Construction and Building Materials. 138, 240-246 https://doi.org/10.1016/j.conbuildmat.2017.01.113 (2017).

13. Cho, J., Jang, H. G., Kim, S. Y. \& Yang, B. Flexible and coatable insulating silica aerogel/polyurethane composites via soft segment control. Composites Science and Technology. 171, 244-251 https://doi.org/10.1016/j.compscitech.2018.12.027 (2019).

14. Pareta, A. S., Gupta, R. \& Panda, S. K. Experimental investigation on fly ash particulate reinforcement for property enhancement of PU foam core FRP sandwich composites. Composites Science and Technology. 195, https://doi.org/10.1016/j.compscitech.2020.108207 (2020).

15. Cao, M. et al. Preparation and performance of the modified high-strength/high-modulus polyvinyl alcohol fiber/polyurethane grouting materials. Construction and Building Materials. 168, 482-489 https://doi.org/10.1016/j.conbuildmat.2018.02.173 (2018).

16. Castellà, N., Grishchuk, S. \& Karger-Kocsis, J. Thermoset polyurea resins with in situ produced silicate filler from water glass: effects of water dispersible alumina nanoparticles. Plastics, Rubber and Composites. 36, 122-127 https://doi.org/10.1179/174328907x177626 (2007).

17. Grishchuk, S., Castellà, N. \& Karger-Kocsis, J. Hybrid resins from polyisocyanate/vinyl ester/water glass systems: Structure and properties. Eur. Polymer J. 43, 1245-1257 https://doi.org/10.1016/j.eurpolymj.2007.01.010 (2007).

18. Karger-Kocsis, J., Castellà, N. \& Grishchuk, S. Hybrid resins from polyisocyanate, vinyl ester, melamine formaldehyde and water glass: structure and properties. Plastics, Rubber and Composites. 37, 204-209 https://doi.org/10.1179/174328908x309349 (2008).

19. Castella, N., Grishchuk, S., Karger-Kocsis, J. \& Unik, M. Hybrid resins from polyisocyanate, epoxy resin and water glass: chemistry, structure and properties. Journal of Materials Science. 45, 1734-1743 https://doi.org/10.1007/s10853-009-4145-9 (2010).

20. Vagenas, N. Quantitative analysis of synthetic calcium carbonate polymorphs using FT-IR spectroscopy. Talanta. 59, 831-836 https://doi.org/10.1016/s0039-9140(02)00638-0 (2003).

21. Awad, W. H. \& Wilkie, C. A. Investigation of the thermal degradation of polyurea: The effect of ammonium polyphosphate and expandable graphite. Polymer. 51, 2277-2285 https://doi.org/10.1016/j.polymer.2010.03.033 (2010).

22. Pashaei, S., Syed, A. A. \& Siddaramaiah \& Thermal Degradation Kinetics of Polyurethane/Organically Modified Montmorillonite Clay Nanocomposites by TGA. Journal of Macromolecular Science, Part A. 47, 777-783 https://doi.org/10.1080/10601325.2010.491756 (2010).

23. Petkowicz, D. I., Canal, S., Finger, P. H., Mignoni, M. L. \& dos Santos, J. H. Z. Synthesis of hybrid zeolites using a solvent-free method in the presence of different organosilanes. Microporous and Mesoporous Materials. 241, 98-106 https://doi.org/10.1016/j.micromeso.2016.11.030 (2017).

24. Castellà, N. Development and Characterization of Polyurea-based Thermoset Resins Entwicklung und Charakterisierung von Polyharnstoff-basierten Harzen, (2009). 
Figures

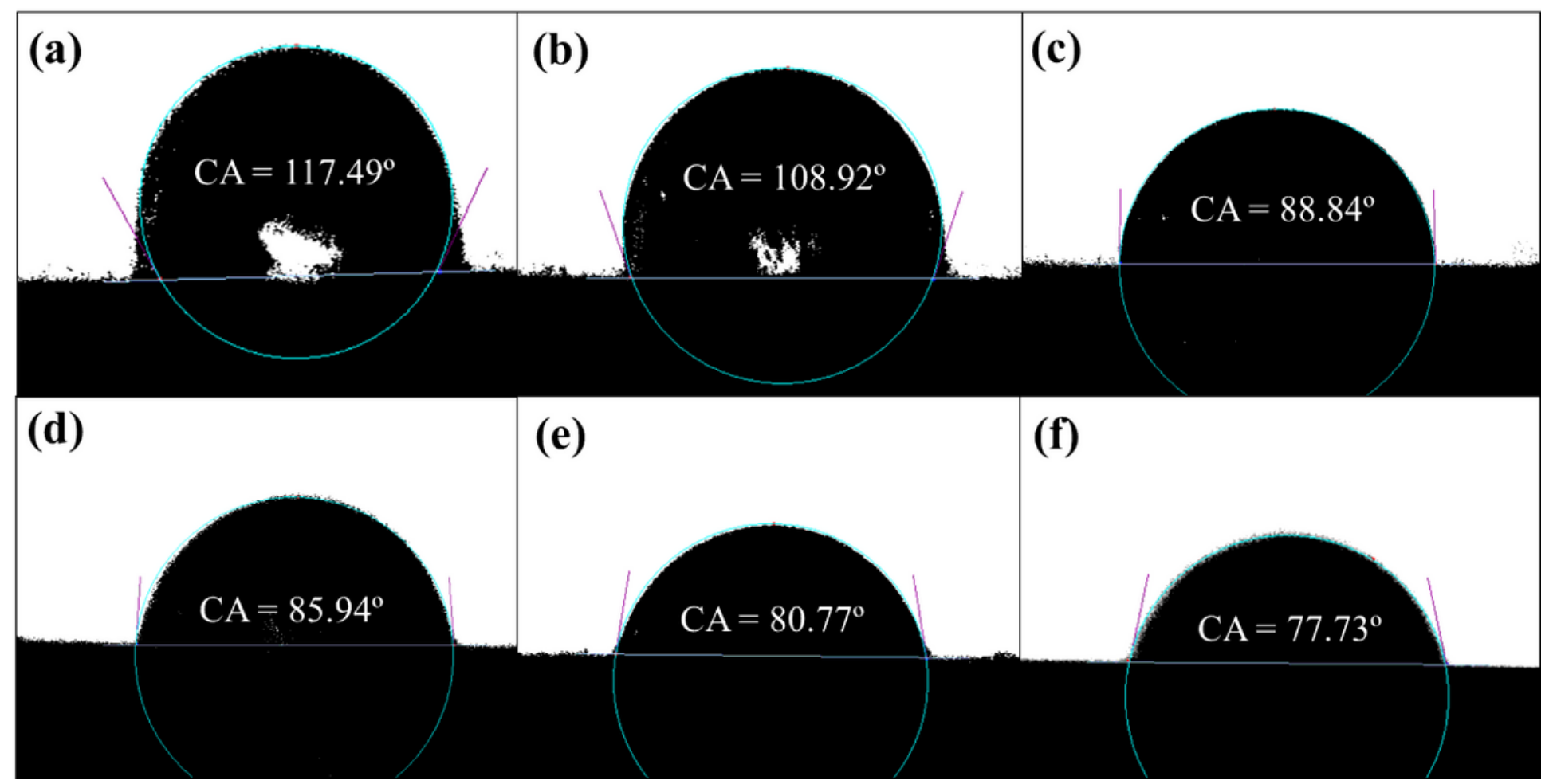

Figure 1

Contact angle images of WG with different CTS content: (a) $0.0 w t \%$, (b) $1.5 w t \%$, (c) $2.0 w t \%$, (d) $2.5 w t \%$, (e) $3.0 w t \%$, (f) $3.5 w t \%$. 


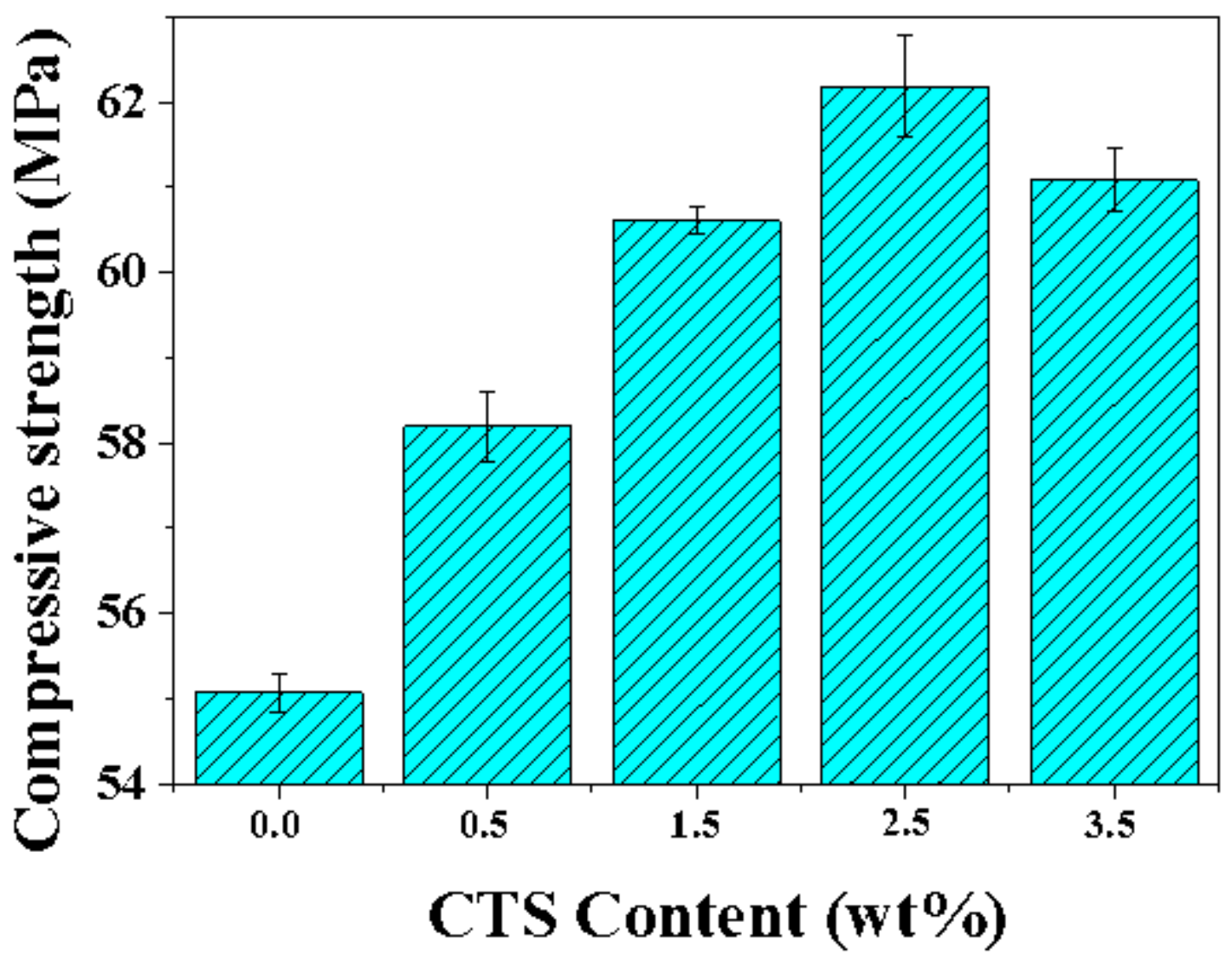

Figure 2

Compressive strength of the WG/PU composites with different CTS contents. 


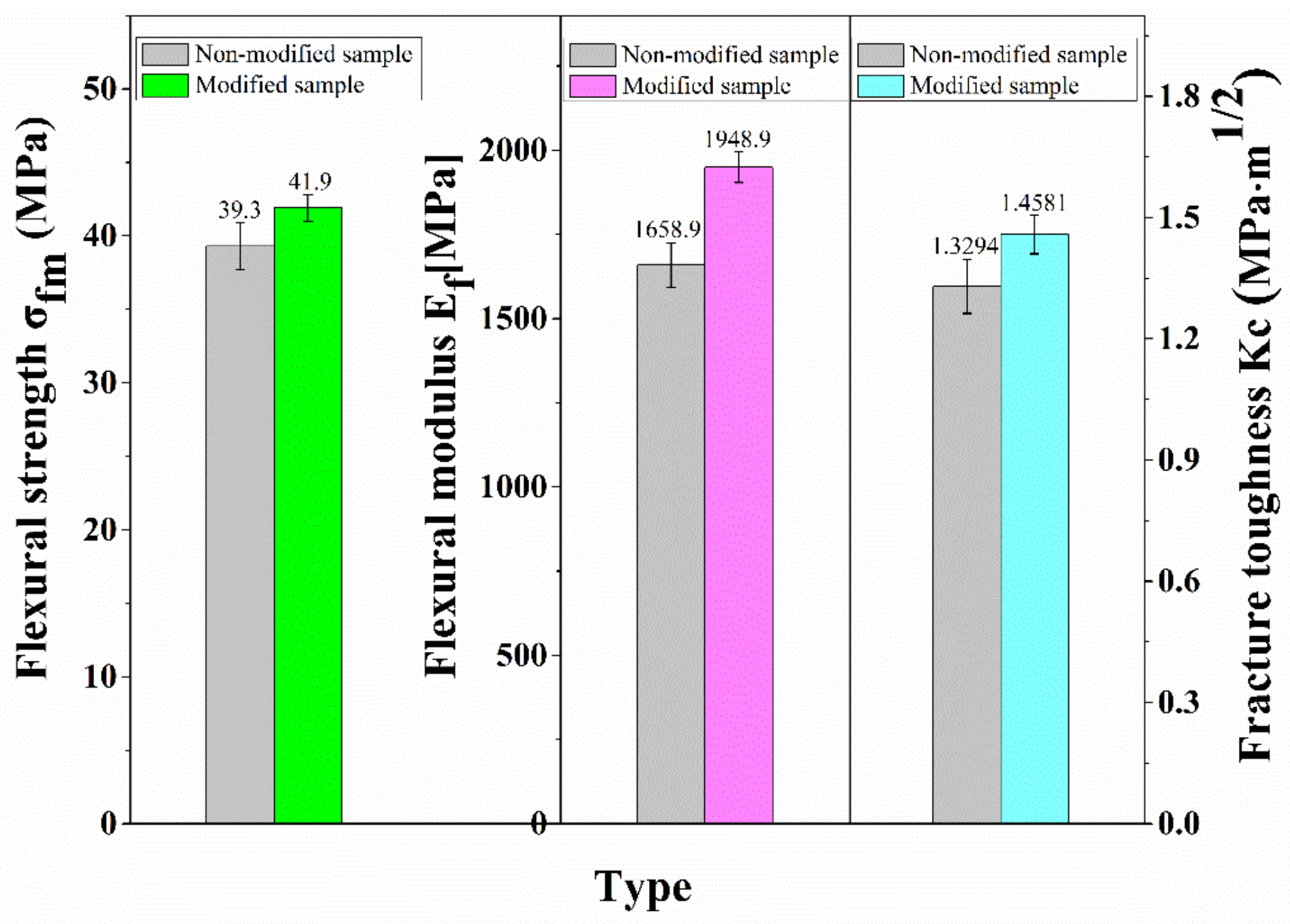

Figure 3

Flexural strength, flexural modulus and fracture toughness of the non-modified and modified WG/PU composites 

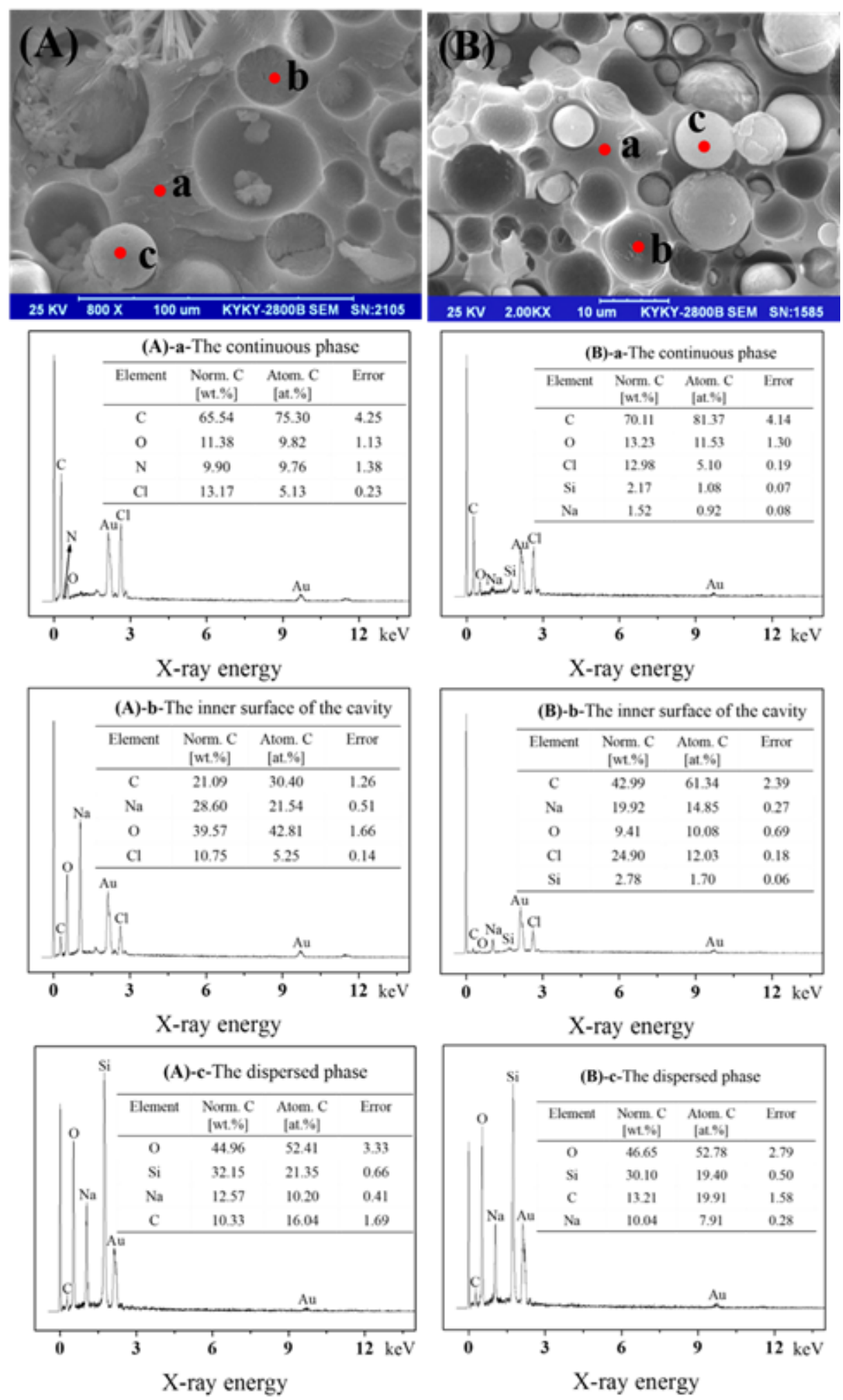

Figure 4

SEM images and EDS spectra of the fracture surface of (A) non-modified WG/PU and (B) modified WG/PU composites (a: the continuous phase, b: the inner surface of the cavity and c: the dispersed phase) 

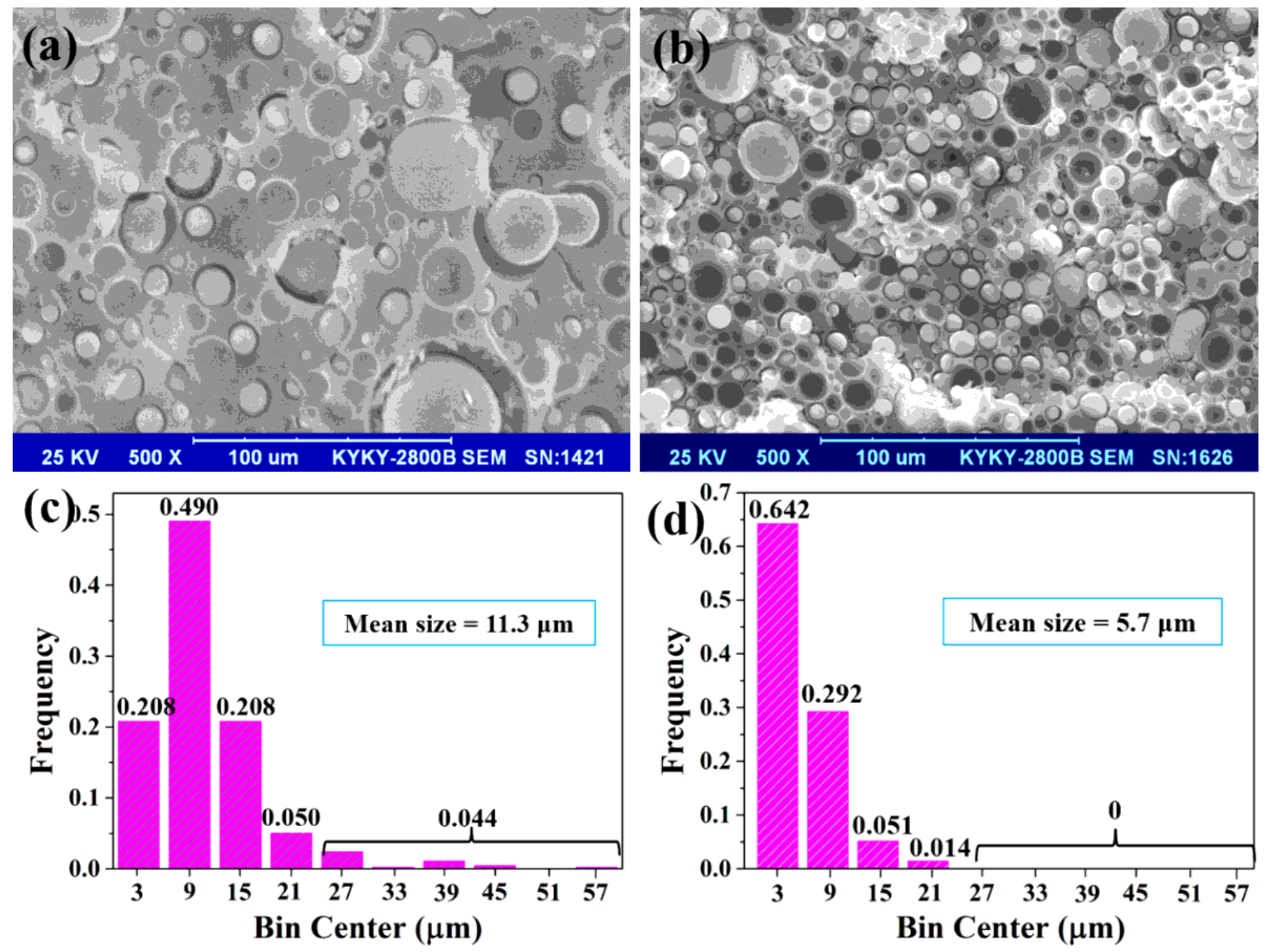

Figure 5

The cross-sectional SEM images of (a) non-modified and (b) modified WG/PU composites; and size distribution histograms of the polysilicate particles in (c) non-modified and (d) modified WG/PU composites. 


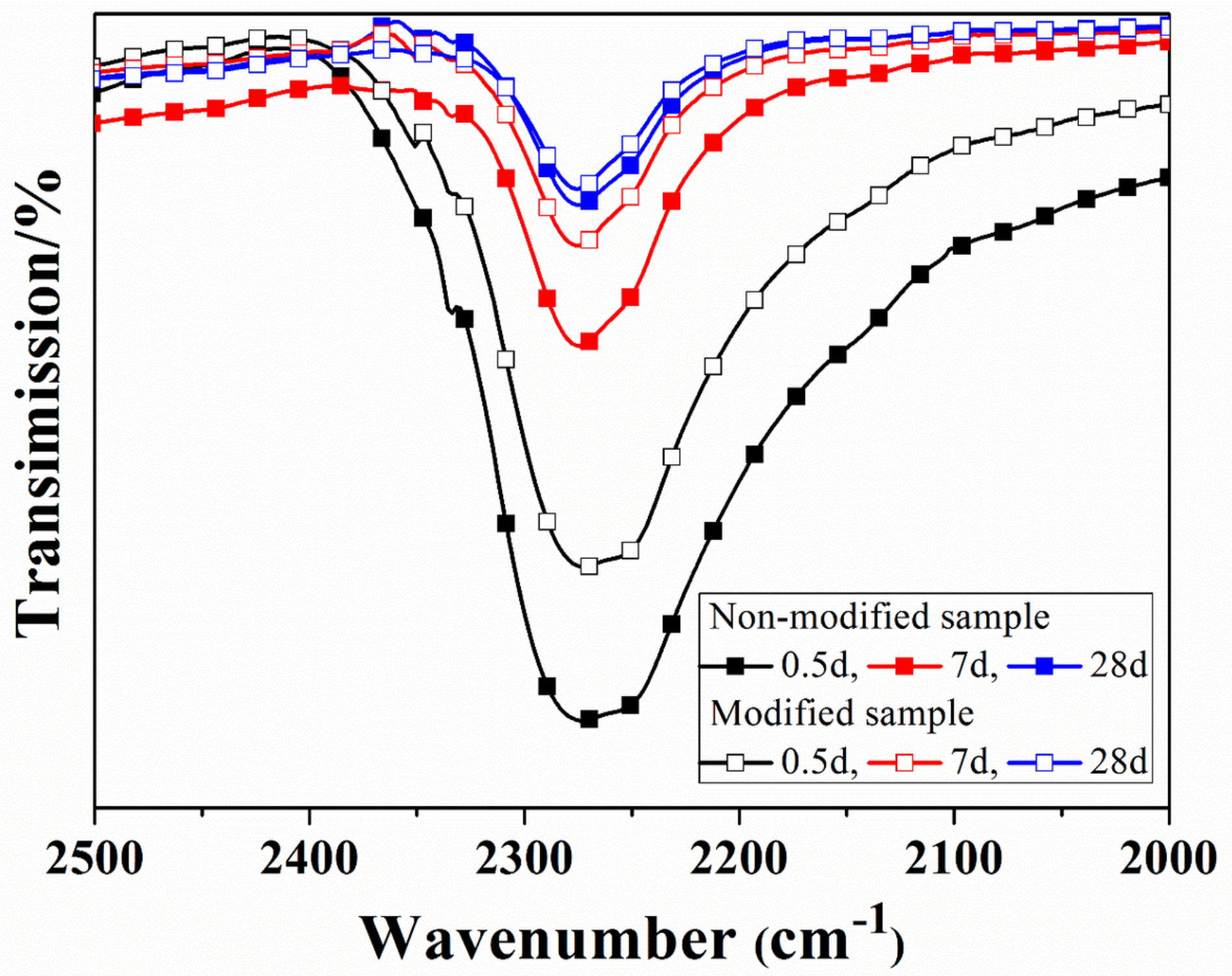

Figure 6

IR spectra of NCO group in the non-modified and modified WG/PU composites during the curing process. 


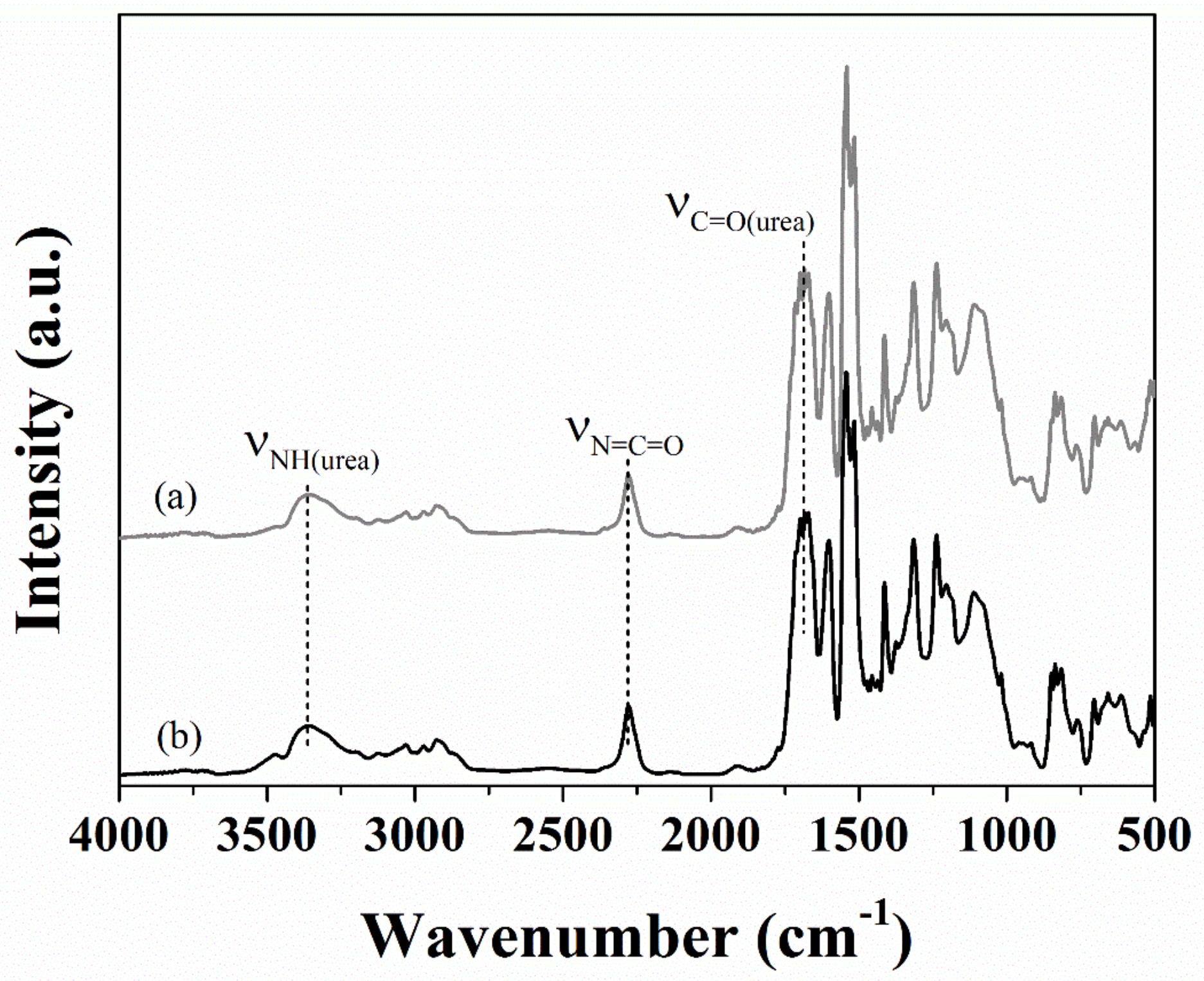

Figure 7

DRIFTS spectra of (a) modified and (b) non-modified WG/PU composites.
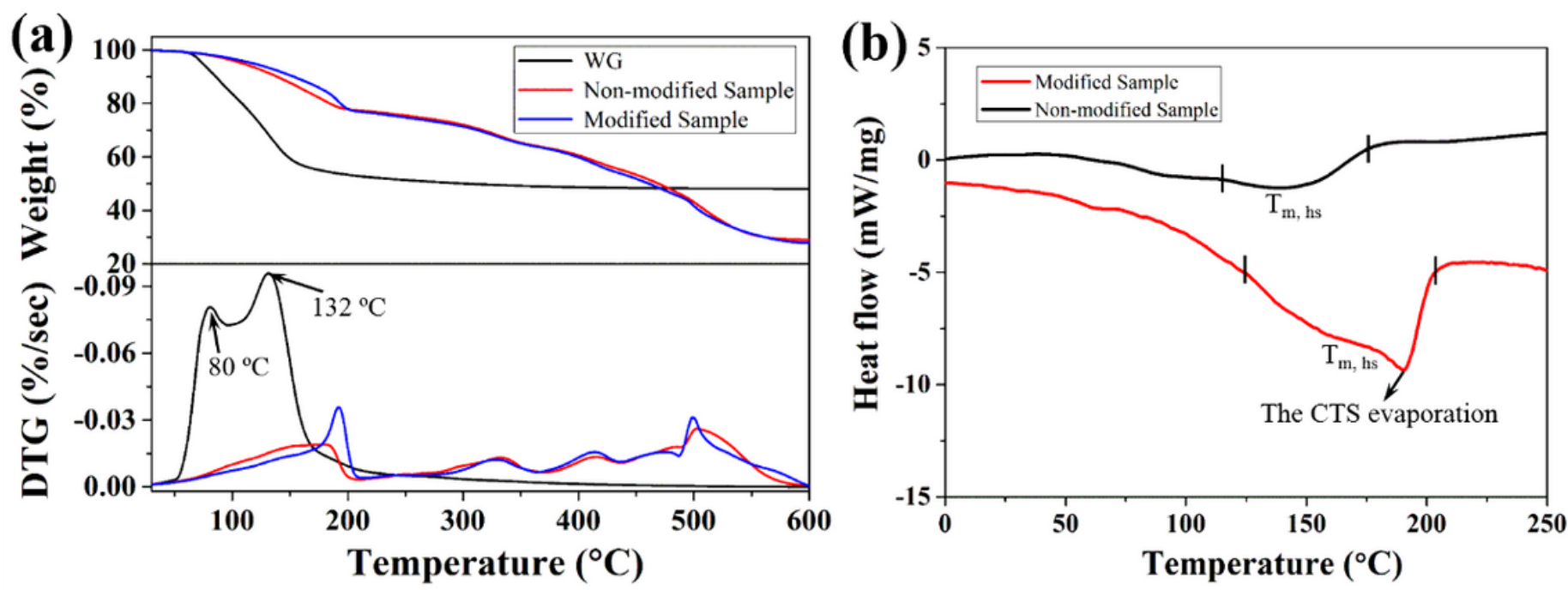
Figure 8

(a) TGA-DTG and (b) DSC curves of WG and WG/PU composites before and after modification.

\section{Supplementary Files}

This is a list of supplementary files associated with this preprint. Click to download.

- Onlinefloatimage1.png 